\title{
Statistical relationship between solar flares and coronal mass ejections
}

\author{
Seiji Yashiro ${ }^{1,2,3}$ and Nat Gopalswamy ${ }^{2}$ \\ ${ }^{1}$ Interferometrics Inc., Herndon, Virginia 20171, USA \\ ${ }^{2}$ NASA Goddard Space Flight Center, Greenbelt, Maryland 20771, USA \\ ${ }^{3}$ The Catholic University of America, Washington, DC 20771, USA \\ email: Seiji.Yashiro@nasa.gov, Nat.Gopalswamy@nasa.gov
}

\begin{abstract}
We report on the statistical relationships between solar flares and coronal mass ejections (CMEs) observed during 1996-2007 inclusively. We used soft X-ray flares observed by the Geostationary Operational Environmental Satellite (GOES) and CMEs observed by the Large Angle and Spectrometric Coronagraph (LASCO) on board the Solar and Heliospheric Observatory (SOHO) mission. Main results are (1) the CME association rate increases with flare's peak flux, fluence, and duration, (2) the difference between flare and CME onsets shows a Gaussian distribution with the standard deviation $\sigma=17 \mathrm{~min}(\sigma=15 \mathrm{~min})$ for the first (second) order extrapolated CME onset, (3) the most frequent flare site is under the center of the CME span, not near one leg (outer edge) of the CMEs, (4) a good correlation was found between the flare fluence versus the CME kinetic energy. Implications for flare-CME models are discussed.
\end{abstract}

Keywords. Sun: flares, Sun: coronal mass ejections (CMEs)

\section{Introduction}

A solar flare is an explosion in the solar atmosphere observed as a sudden flash of electromagnetic radiation emitted from the heated plasma. A coronal mass ejection (CME) is an eruption of magnetized plasma from the Sun into the interplanetary medium. After the discovery of CMEs in 1971, the relationship between them has been examined extensively (see Kahler 1992 for review). Munro et al. (1979) reported that $\sim 40 \%$ of $\mathrm{CMEs}$ were associated with $\mathrm{H} \alpha$ flares. Andrews (2003) reported that $\sim 60 \%$ of M-class flares were were associated with CMEs. There is no one-to-one flare-CME occurrence (Harrison 1995). However, the similarity between the derivative of the X-ray light curve and CME accelertion profile (Zhang et al. 2001, Vršnak et al. 2004) suggest a close relation. Both phenomena are thought to be different manifestations of the same process (Harrison 1995, Jing et al. 2005).

The Large Angle and Spectrometric Coronagraph (LASCO; Brueckner et al. 1995) on board the Solar and Heliospheric Observatory (SOHO) mission has observed more than 13,000 CMEs from 1996, providing a great opportunity to investigate the flareCME relationship. In this paper we report the statistical relationship between flares and CMEs.

\section{Flare-CME association}

Yashiro et al. (2006) examined the CME association rate as a function of the flare parameters. We repeated that study to include the flare-CME pairs in 2006 and 2007 and restrict the study to limb events. Solar flares have been routinely detected by the 

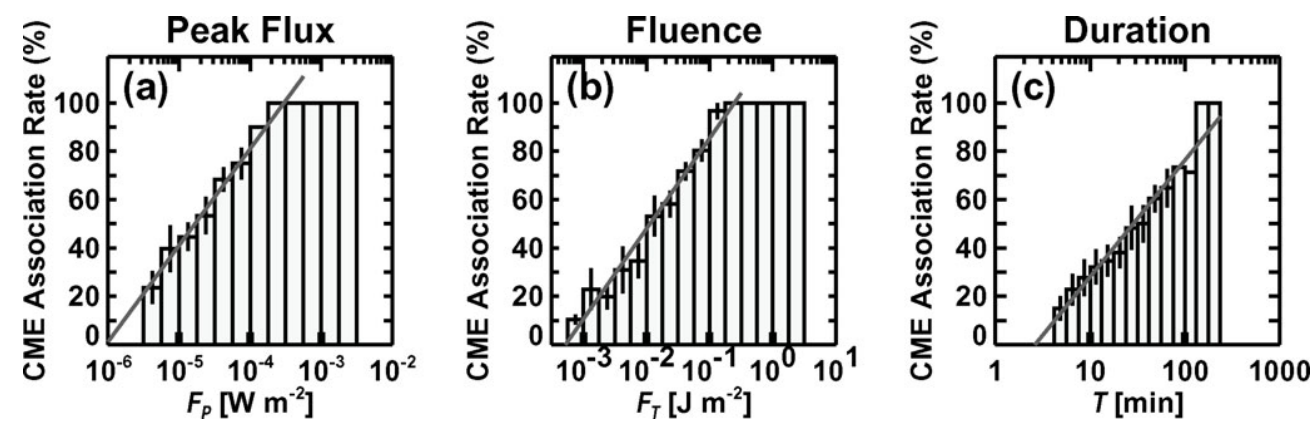

Figure 1. CME association rate as a function of (a) X-ray peak flux $F_{P}$, (b) fluence (total flux) $F_{T}$ and (c) duration $T$. The plots are similar to Fig. 1 of Yashiro et al. 2006, but different data sets were used. See text for details.

X-ray Sensor (XRS) on board the Geostationary Operational Environmental Satellite (GOES) in the two wavelength bands (0.5-4 $\AA$ and 1-8 $\AA$ ). The basic flare parameters are available in the Solar Geophysical Data (SGD) and online Solar Event Reports provided by NOAA. We excluded flares corresponding to SOHO/LASCO downtimes by requiring that at least two LASCO C2 images were obtained between $0-2$ hours after the flare onset. Yashiro et al. (2005) reported that about half of disk CMEs associated with C-class flares and $\sim 16 \%$ of disk CMEs associated with M-class flares were invisible to LASCO. In order to examine the flare-CME relationship properly, we used only limbward events with longitudes $>45^{\circ}$. We also eliminated flares at longitudes $>85^{\circ}$ because of the possible partial occultation of the X-ray source, resulting in an underestimate of the X-ray flux. There are 6374 flares (above C3 level) listed in SGD, but the locations are not listed for $\sim 2000$ of them. For the X- and M-class flares, we identified their locations using solar disk images obtained in X-ray, EUV, $\mathrm{H} \alpha$, and microwave. For C-class flares, we used only those flares with their locations listed in SGD.

We used the SOHO/LASCO CME Catalog (Yashiro et al., 2004) to find the flareassociated CMEs within a 3-hour time window. However, because the time window analysis by itself could produce false flare-CME pairs, we checked the consistency of the associations by viewing both flare and CME movies available in the Catalog. Eruptive surface signatures, such as filament eruptions and coronal dimmings, helped ascertain the associations. However, in some cases, we could not determine with confidence whether the association was true or not because not all flares had clear eruptive signatures. We abandoned to give a clear true or false answer of the CME association of such events, and left them as ambiguous associations. This way, we classified all the flares into three categories: flares with definite CME association, flares with uncertain CME association, and flares that definitely lacked CMEs.

Figure 1 shows the CME association rate as a function of X-ray peak flux (a), fluence (b), and duration (c). The CME association rate has an error range obtained from the uncertain flare-CME pairs. Assuming that all of the uncertain events were false, the lower limit of the CME association was determined by dividing the number of definitive events by the total number of flares. Similarly we obtained the upper limit by assuming that all uncertain events were true. We used the middle of the lower and upper limits as the representative association rate. This is equivalent to assuming that half of the uncertain events had true CME association.

It is clear that the CME association rate of X-ray flares increased with their peak flux, fluence, and duration. The larger flares tend to be associated with the CMEs, but for 
even X-class flares about 10\% of them lacked CME association (Gopalswamy et al., this volume). Figure $1 \mathrm{c}$ is consistent with the well known fact that long duration (or decay) events (LDEs) are well associated with the CMEs, while impulsive flares are not (Sheeley et al. 1983). However, even some impulsive flares with duration $<10 \mathrm{~min}$., about $20 \%$ of them, have associated CMEs (Kahler et al. 1989). There is no critical duration dividing flares into those with and without CMEs.

\section{Temporal relationship}

The onset time is the most basic parameter to investigate the relation between flares and CMEs. Onset times of CMEs are difficult to identify since they originate below the LASCO C2 field of view (FOV). Exceptions are the CMEs observed by LASCO C1 (e.g. Gopalswamy \& Thompson 2000; Zhang et al. 2001). These studies showed that the onsets of the flares and CMEs are tightly connected. However, its data are not available for the most of the CMEs, so the CME onset is either estimated from CME-related surface activities (e.g., filament eruptions) or is extrapolated to the solar surface from the CME trajectory in the LASCO C2 and C3 FOVs. We attempt to examine the temporal relationship using the extrapolated CME onset.

The extrapolated CME onset has been used from pre-SOHO era. The extrapolation method and its weakness were described in many literatures (e.g., Harrison \& Sime 1989, Harrison et al. 1990). We need to make at least two assumptions to obtain the extrapolated onsets. The first is the shape of CME trajectory under the occulting disk. We assume that the unseen trajectory is the same as observed in the LASCO FOVs. In the case of CMEs with constant speed or deceleration motion, the above assumption is apparently incorrect since all CMEs must have an acceleration phase during their initiation. Ignoring the acceleration phase, the extrapolated onsets would be later than the actual onsets. The second necessary assumption is regarding the position where the CME is initiated. We assume that the apex of the CMEs starts from 1 Rs (solar limb). Flare position is a good proxy, but the spatial size of CMEs at the beginning would be larger than that of flares. Ignoring the spatial size of the CMEs, for the limb events, the extrapolated onsets would be earlier than the actual CME onsets.

Figure 2a illustrates how we extrapolate a CME trajectory to obtain the onset. The height-time data are fitted by first-order (dotted line) or second-order (dashed line) polynomials, which correspond to constant speed and constant acceleration (or deceleration), respectively. The extrapolated CME onsets are determined as the time when the fitting lines cross the $1 \mathrm{Rs}$ (horizontal line). $T_{1}$ and $T_{2}$, respectively indicate the onset time determined from first- and second-order polynomials. The linear lines invariably cross 1 Rs, but the parabolic fits do not necessarily do so (Fig. 2b). Figure 2b is an example showing the parabola does not cross 1 Rs line.

The extrapolated CME onsets may not be accurate due to errors resulting from the assumptions stated above. A good example is the flare-CME event on 2002 April 21 (Fig. 2c). An X1.5 flare started at 00:43 UT and then peaked at 01:51 UT. The flareassociated CME was observed by LASCO at 01:27 UT with a speed of $2393 \mathrm{~km} / \mathrm{s}$. The extrapolated CME onset is 01:16 UT for the both linear and parabolic fits $\left(T_{1}\right.$ and $T_{2}$ ). The X1.5 flare started 33 minutes earlier than the associated CME. However, a detailed analysis with TRACE and UVCS observations showed that the eruptive feature associated with the CME started at 00:48 UT at the latest. The difference with the extrapolated CME onset $(28 \mathrm{~min}$ ) should be considered as an error resulting from the assumption that the CME trajectory under the occulting disk is the same as the observed one in the LASCO FOV. Another example is a flare-CME event on 1998 January 25 

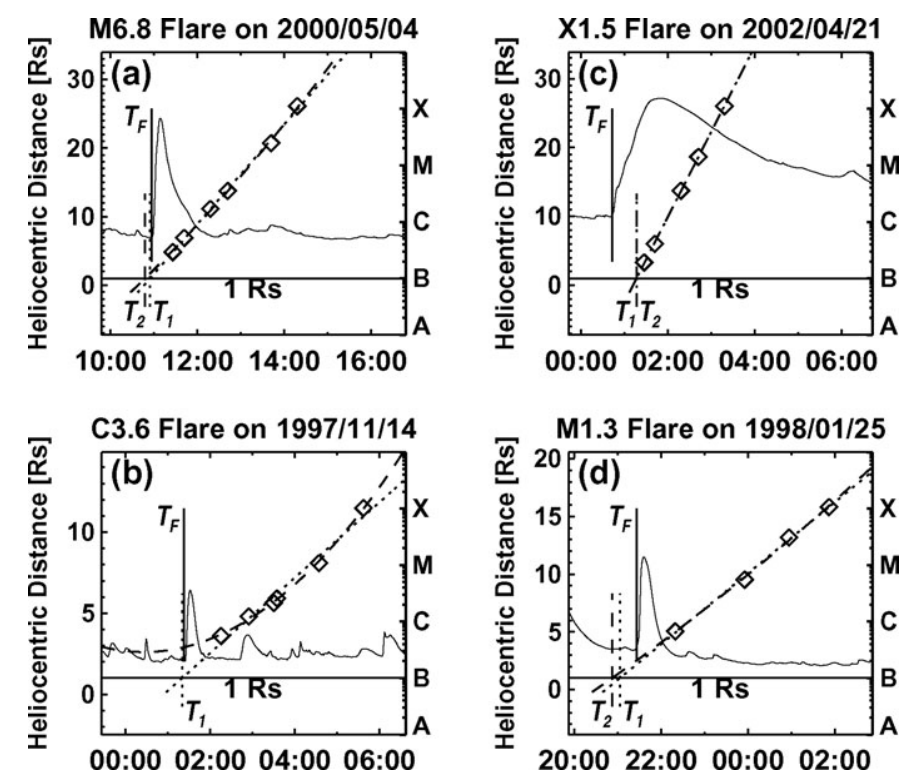

Figure 2. CME Height-time diagram superposed with X-ray light curve to illustrate how we estimate the CME onset. Solid curves are X-ray intensity obtained by GOES satellite (right scale), and $T_{F}$ indicates flare onset recorded in NOAA/SGD. Diamonds shows heliocentric distance of the CMEs, which are recorded in the CME Catalog. Dotted lines and dashed curves are first- and second-order polynomial fitting, respectively.
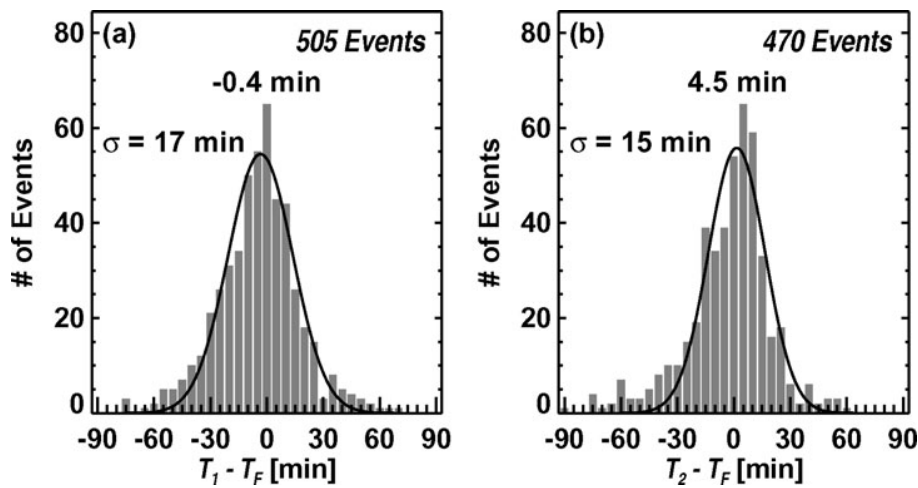

Figure 3. Distributions of onset difference between flares and CMEs. The CME onsets are estimated by (a) first-order and (b) second-order polynomial fitting to the CME height-time data.

(Fig. 2d). An M1.3 flare started at 21:26 UT and the associated CME appeared in C2 FOV at 22:19 UT. In this case, the CME height-time measurements missed the deceleration motion during the CME initiation.

Figure 3 shows the distributions of the difference between the flare and CME onsets. We used only definitive flare-CME pairs for this analysis. The CME onsets $\left(T_{1}\right.$ and $T_{2}$ ) are estimated using linear and parabolic fitting, respectively, as described above. Both distributions are well represented by Gaussians. The parabolic fit must return a better result to the CME trajectory because it has a higher degrees of freedom. The standard deviation is $17 \mathrm{~min}$ for linear and $15 \mathrm{~min}$ for parabolic fits. Gopalwamy et al. 

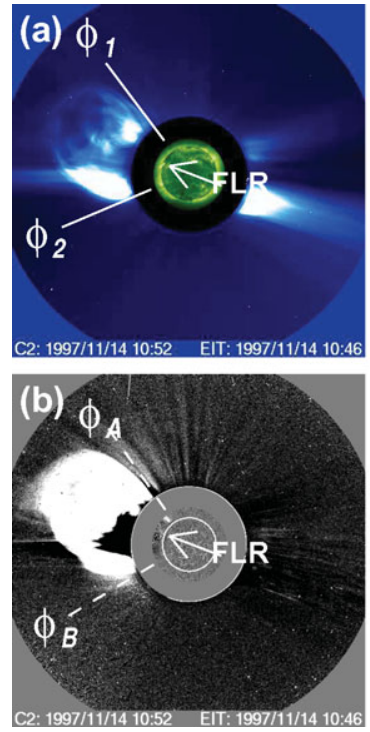
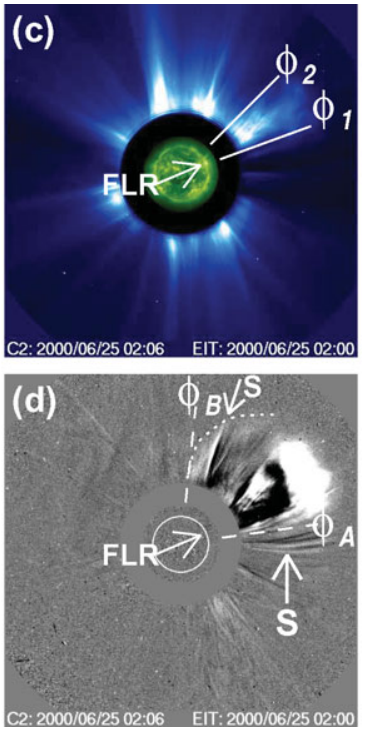
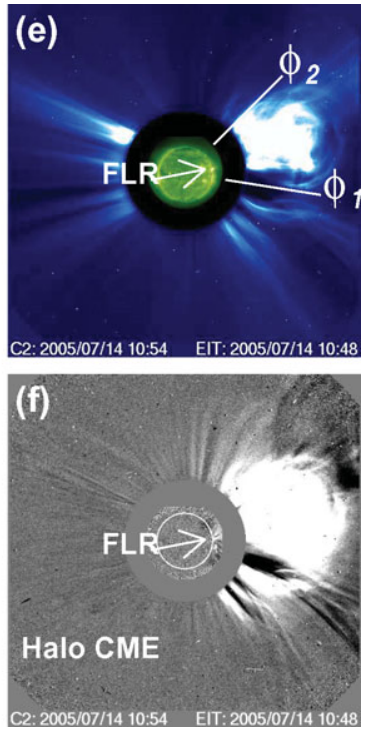

Figure 4. Three CMEs observed by SOHO LASCO to illustrate the measurement of CME span. The top row shows direct images used to measure the main CME body, and the bottom row shows corresponding running difference images used to measure the whole CME. The side edges of the main CME body (the whole CME) are denoted by $\phi_{1}$ and $\phi_{2}\left(\phi_{A}\right.$ and $\left.\phi_{B}\right)$. Arrows point to the position of the flares associated with the CMEs (from Yashiro et al. 2008).

(2003) examined the onset times of the prominence eruptions and CMEs and found similar distribution. Since the prominence eruptions and CMEs are parts of the same phenomenon, their onsets should be the same. Therefore it is reaasonable to consider that the differnce between the flare and extrapolated CME onsets results not from the nature of flare-CME relation but from the error of the extrapolated CME onset.

\section{Spatial relationship}

The spatial relationship between flares and CMEs has been investigated from the 1970s and 1980s with the CME observations obtained by the Solwind and the Solar Maximum Mission (SMM) coronagraphs. Harrison (1986) analyzed 48 flare-CME events observed by SMM and Solwind and reported that many flares occurred near one leg of the associated CMEs (see also Harrison 1991, 1995). Kahler et al. (1989) examined 35 events observed by Solwind and reported that flare positions did not peak either at the center or at one leg of the CMEs. However recently Yashiro et al. (2008) examined 498 events observed by LASCO from 1996 to 2005 and found that the most probable flare site is at the center of the CME span. Here we extended that study to include the flare-CME pairs in 2006 and 2007.

First we briefly describe the analysis of Yashiro et al. For each CME, the angular widths of two structures were measured. One is the main CME body which corresponds to the three-part structure of a CME. The other is the whole CME. Some CMEs possess a faint envelope outside of the main CME body. The envelope structure could be a shock driven by the CME hence not a part of the CME. However, since there is no established way to identify a shock by coronagraph observation itself, we have included the envelope structures as a part of the CME and refer to all the CME features as the whole CME. Figure 4 illustrates how we measured the PAs of the main CME body and the whole 
CME. The CME on 1997 November 14 (Figs. 4a and 4b) did not have an envelope, thus the $\phi_{1}\left(\phi_{2}\right)$ and $\phi_{A}\left(\phi_{B}\right)$ are identical. The angular width is defined as the difference of the two outer edges $\left(\phi_{2}-\phi_{1}\right.$ for the main CME body and $\phi_{B}-\phi_{A}$ for the whole CME). Note that the angular width of a CME would be affected by projection effects if the CME originated away from the solar limb. The measrued CME widths are systematically larger than their true values (See Burkepile et al. 2004). The CME on 2000 June 25 had a faint envelope to the north of the main CME body (Figs. 4c and 4d). The northern edge of the envelope denoted by $\phi_{B}$ is used for the edge of the whole CME. Since we cannot see an envelope to the south of the CME, the southern edges of the main CME body and whole CME are almost identical. The CME on 2005 July 14 appeared in the C2 FOV at 10:54 UT (Figs. 4e and 4f). The CME had a clear three-part structure with a faint envelope. The envelope covered the occulting disk at 11:54 UT, thus the CME is listed as a halo (Howard et al. 1982) in the CME catalog. In this case $\phi_{A}$ and $\phi_{B}$ cannot be determined.

The distributions of differences between flare PAs and CME CPAs for the main CME body and for the whole CME are shown in Figures 5 a and 5e, respectively. Both the distributions are very similar and are well represented by Gaussians. The standard deviation is $17.2^{\circ}$ for the difference between the flares and main CME bodies and $17.5^{\circ}$ for the difference between the flares and whole CMEs. We separated the events into three groups according to their flare intensity and made the same plots for each group. The second, third, and forth rows in Figure 5 correspond to the events with X-class, M-class, and $\mathrm{C}$-class flares, respectively. The standard deviation is shown in each plot, which ranges from $17.1^{\circ}-17.7^{\circ}$ for the main CME body and $16.8^{\circ}-21.1^{\circ}$ for the whole CME.

In order to investigate the flare position with respect to the main CME body (frontal structure), we normalize the PA differences by the half angular span and show its distribution in Figure 5i. It is clear that most of the flares are located under the span of the main CME body. Figure $5 \mathrm{~m}$ is the same as the Figure 5i, but for flare locations with respect to the edges of the whole CMEs. Both the distributions are well represented by Gaussians with standard deviations of 0.59 for the main CME body and 0.38 for the whole CME. In both the cases, the peak of the Gaussian is around zero, meaning that the flares frequently occur under the center of the CME span, not near one leg (outer edge) of the CMEs. Then we separated the events into three groups according to their flare intensity. We found that all distributions have a peak around zero, while the width of the distributions is different for different flare levels. The flare-CME events with Xclass flares (hereafter X-class events) have a narrower distribution suggesting that many $\mathrm{X}$-class flares lie under the center of the CME span. On the other hand, the C-class events have a broader distribution and a significant number of events occurred outside of the CME span.

\section{Energy relationship}

Figures 6 and 7 show scatter plots of logarithms of flare and CME parameters. The flare parameters in the X-axis are peak flux (left), fluence (center), and duration (right). The CME parameters in the Y-axis are speed (Fig. 6; top), main body width (Fig. 6; middle), whole CME width (Fig. 6; bottom), mass (Fig. 7; top), and kinetic energy (Fig. 7; bottom). The definition of the main body and whole CME is described in Section 4 . The correlation coefficients of the logarithms of these parameters are shown on the plots.

The familiar parameters representing flare and CME properties are peak soft X-ray flux and speed, respectively. The former has been used to determine the rank of solar flares. The scatter plot of these parameters shows that a correlation exists between them, 
PA Difference
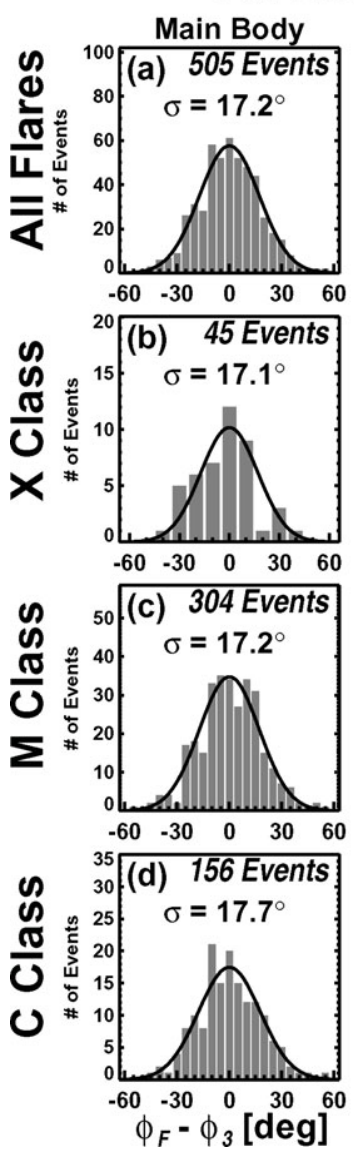

Whole CME
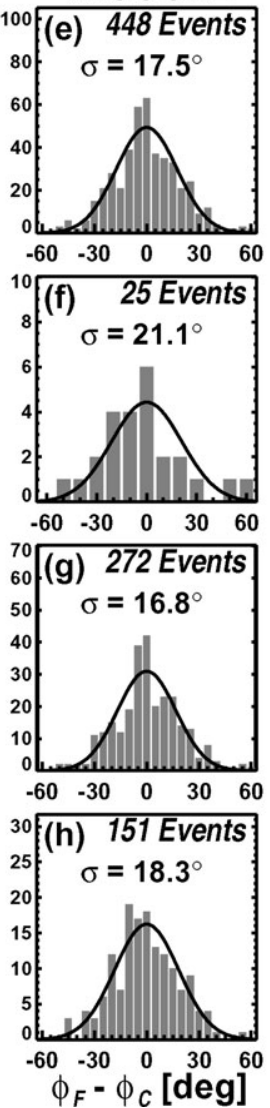

Relative Position

Main Body

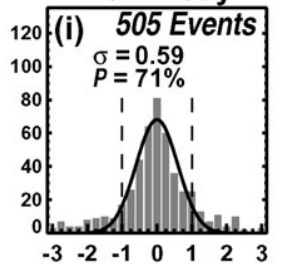

Whole CME
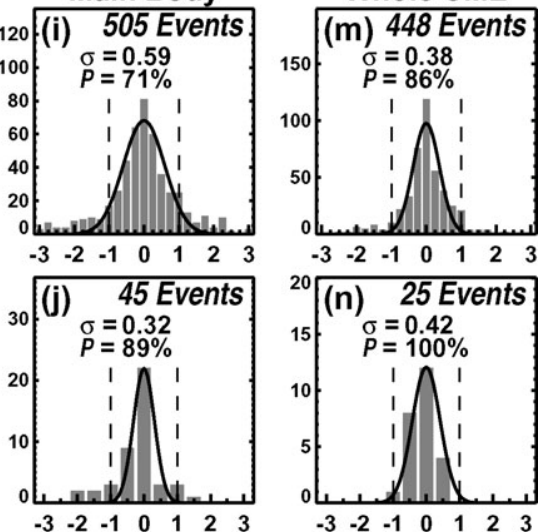

$\sqrt[20]{(\mathrm{n}) \quad 25 \text { Events }}$
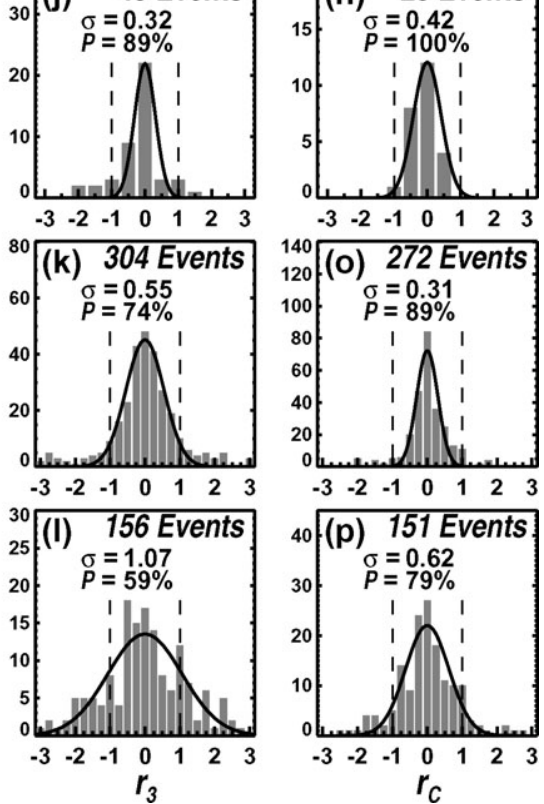

Figure 5. Distribution of flare positions with respect to the CPA of the CME. The first and second colums show the difference of the flare PAs $\left(\phi_{F}\right)$ and CME CPAs $\left[\phi_{3}=0.5\left(\phi_{1}+\phi_{2}\right)\right.$; $\left.\phi_{C}=0.5\left(\phi_{A}+\phi_{B}\right)\right]$. The standard deviation obtained by the Gaussian fit is shown in each plot. The third and fourth columns show the distributions of PA difference normalized by the half-CME span $\left[r_{3}=\left(\phi_{F}-\phi_{3}\right) / 0.5\left(\phi_{2}-\phi_{1}\right) ; r_{C}=\left(\phi_{F}-\phi_{C}\right) / 0.5\left(\phi_{B}-\phi_{A}\right)\right]$. The vertical dashed lines mark two side edges of the CMEs. $P$ is the percentage of flares lying inside of the CME span. The second, third, and fourth rows correspond to the event with X-class, M-class, and C-class flares, respectively (from Yashiro et al. 2008; Extended data set).

i.e. larger flares are associated with faster CMEs, but the correlation coefficient is not high (0.50). The scatter is wider for weaker flares. For C-class flares, the speed of the associated CMEs ranges from $146-1378 \mathrm{~km} / \mathrm{s}$ (an order of magnitude), while 882 $3387 \mathrm{~km} / \mathrm{s}$ (approximately a half order of magnitude) for the largest flares (>X3 level). A better correlation (0.56) is found in the flare fluence (total flux) versus CME speed. Moon et al. (2002) reported a correlation coefficient of 0.47 between flare fluence and CME speed observed by GOES and SOHO/LASCO. The coefficient is lower than ours (0.56). Since their data period is from 1996 to 2000, their sample did not have many extreme events. (Out of 13 huge flares (> X3.0 level) in solar cycle 23, ten occurred after Jan 2001). The correlation between flare duration and CME speed is poor $(\mathrm{r}=0.23)$. This very weak correlation could be the result of a chain of correlations, since the duration is correlated with the fluence $(\mathrm{r}=0.68$; Veronig et al. 2002). 

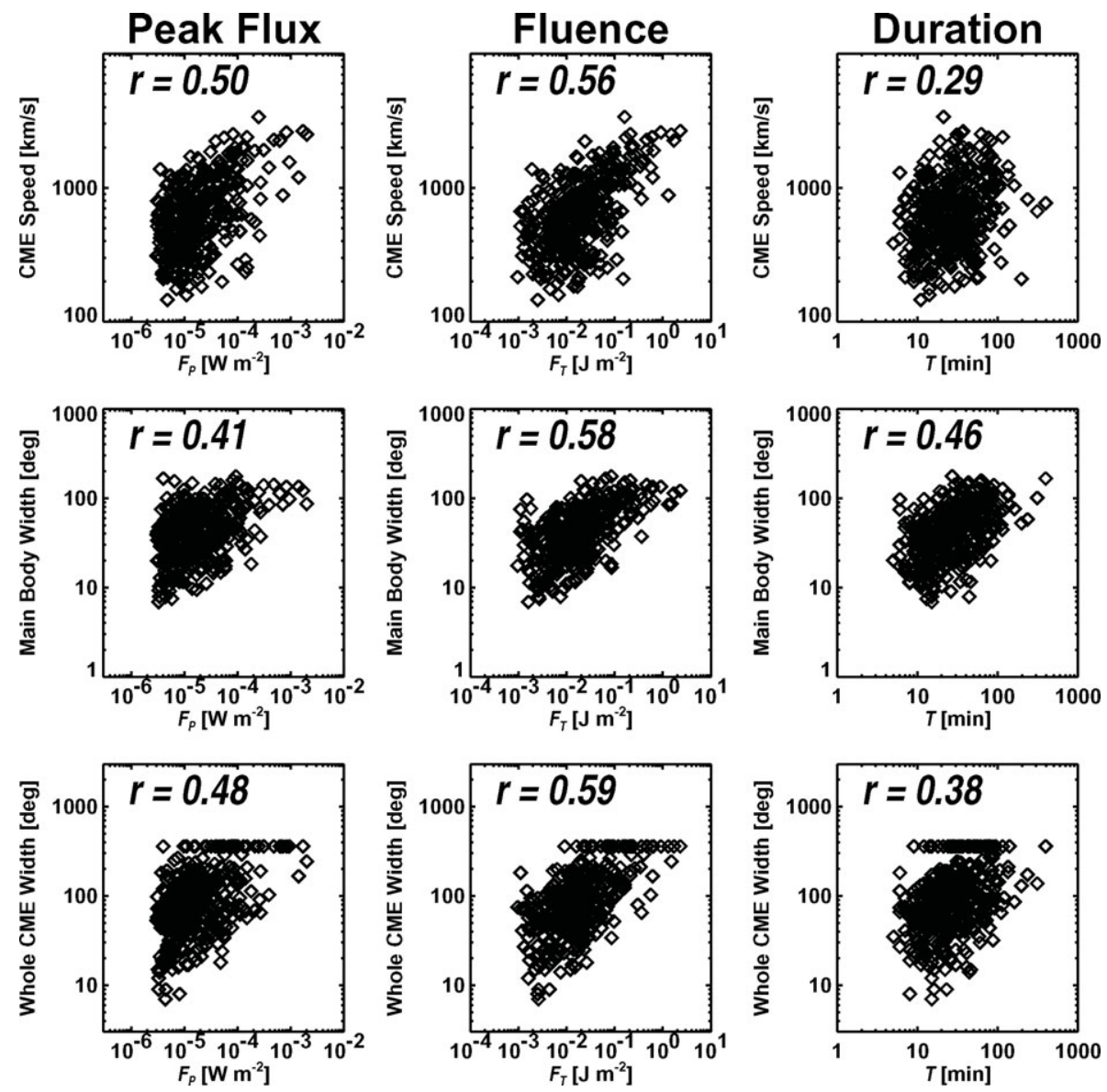

Figure 6. Scatter plots between flare and CME parameters. The $\mathrm{X}$ axis is for flare parameters, peak flux (left), fluence (center), and duration (right) and the Y axis is for CME parameters, speed (top), main body width (middle), and whole CME width (bottom). See Figure 4 for the definition of the main body width and whole CME width. The correlation coefficient is shown on the plot.

Since the determination of CME widths is controversial (see Section 4), for each CME we measured angular width of two structures: main body and whole CME. The main body corresponds to the CME three-part structure. The whole CME includes a faint envelope occasionally seen outside of the main body. The evelope could be a shock driven by the CME. If this is the case, the envelope is not a part of the CME. Although we used both widths to investigate the correlation with flare parameters, the scatter plots do not show significant differences between them. Similar to the CME speed, the best correlation is found in the flare fluence versus the CME width. Kahler et al. (1989) reported that the correlation coefficient between the CME width and the logarithms of flare duration is 0.67 , which is higher than ours (0.46). We should note here that the definitions of the flare duration are different. Kahler et al. determined the end of the flare as the time when the X-ray flux returns to the GOES C2 level. Our flare end corresponds to the time when the soft X-ray flux decays to a point halfway between the peak flux and the pre-flare background level. The different definition would explain the different correlation coefficients. 

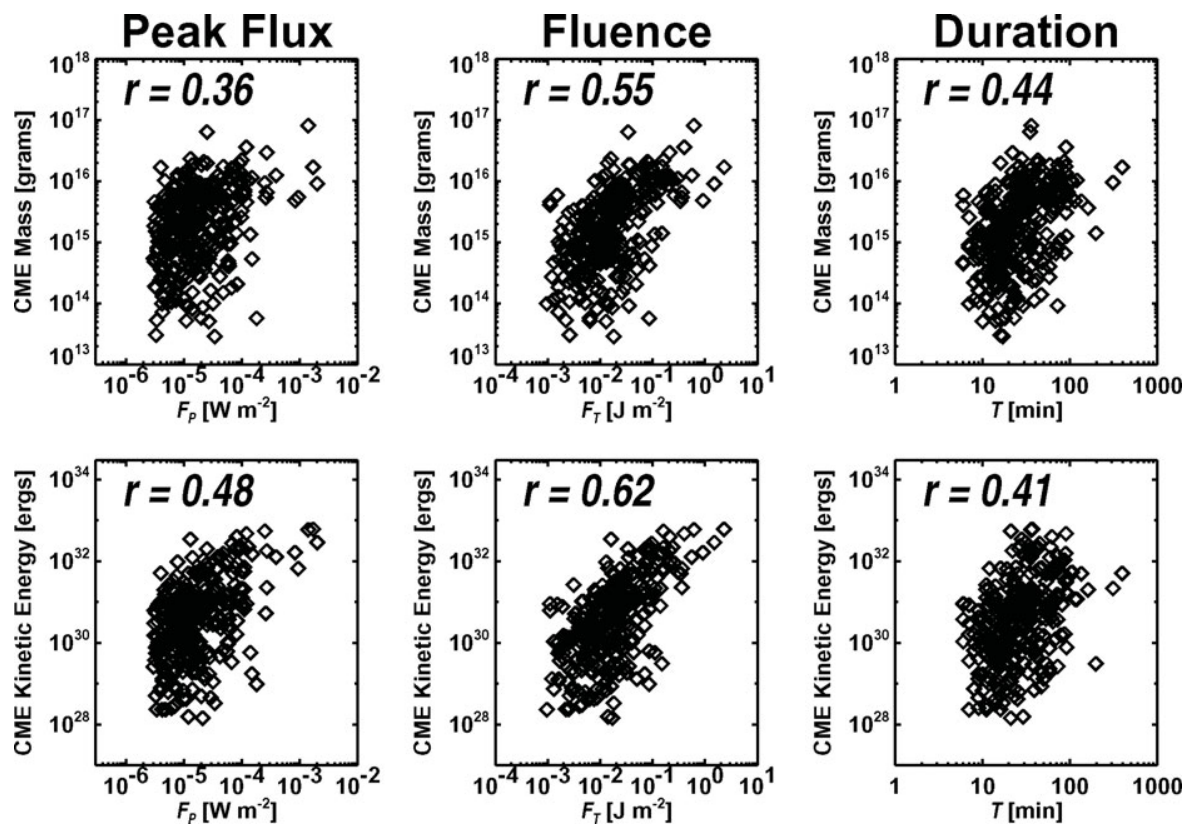

Figure 7. Scatter plots between flare and CME parameters. The $\mathrm{X}$ axis is for flare parameters, peak flux (left), fluence (center), and duration (right) and the $\mathrm{Y}$ axis is for CME parameters, mass (top) and kinetic energy (bottom).

Hundhausen (1997) reported a correlation between the flare peak flux and CME kinetic energy for 249 flare-CME pairs observed by GOES and SMM. The correlation coefficient of logarithms of these parameters is 0.53. By eliminating the disk events to avoid projection effects, Burkepile et al. (2004) obtained a better correlation of 0.74. The former is comparable to ours (0.48) but the latter is significantly higher. For the C-class flares $\left(10^{-6} \leqslant F_{P}<10^{-5} \mathrm{~W} \mathrm{~m}^{-2}\right)$, the kinetic energy of the associated CMEs ranges over nearly 4 orders of magnitude $\left(10^{28}-10^{32}\right.$ ergs $)$ in our plot, while only over 2 orders of magnitude $\left(10^{29.5}-10^{31.5}\right.$ ergs $)$ in Burkepile et al. plot. It is possible that the higher correlation coefficient obtained by Burkepile et al. is due to a smaller number of events (24).

\section{Discussion \& summary}

CME observations by SOHO LASCO over the whole solar cycle enabled us to perform an extensive statistical analysis of flare-CME relationships. We examined the CME associations of flares from 1996 to 2007 and found that the CME association rate clearly increases with the flare's peak flux, fluence, and duration.

Case studies on the flare-CME initiation revealed that the onsets of the flares and CMEs are tightly connected (Zhang et al. 2001, Vršnak et al. 2004). We attempted to confirm this for a large number of events, but the extrapolated CME onsets are not accurate enough to examine the initiation process. The difference between the flare and CME onsets shows symmetrical Gaussian distributions with standard deviations $\sigma=17 \mathrm{~min}(\sigma=15 \mathrm{~min}$ ) for the first (second) order extrapolated onset. We conclude that the difference between the flare and extrapolated CME onsets results from the error of the extrapolated CME onset. 
Pre-SOHO studies reported that many flares occurred near one leg of the associated CMEs (Harrison et al. 1986), or there is no preferred site with respect to the CME (Kahler et al. 1989). Yashiro et al. (2008) examined the spatial relationship between the flares and CMEs and found that the most probable site is at the center of the CME span. The result is suitable for flare-CME models typified by the CSHKP (CarmichaelSturrock-Hirayama-Kopp-Pneuman) reconnection model. They also reanalyzed the preSOHO data and concluded that the long-term LASCO observation enabled us to obtain the detailed spatial relation between flares and CMEs.

The highest correlation coefficient was found in the scatter plot between the flare fluence and CME kinetic energy $(\mathrm{r}=0.62)$. Note that the fluence of a flare, which is obtained by integrating the X-ray flux from its start to end, have been used as a proxy of the flare radiation energy. Their energetic connection is tighter for larger events. For a given Cclass flare, the kinetic energy of assocaited CME ranges over 3 orders of magnitude, while under 2 orders of magnitude for a given X-class flare. This would be related to our finding on the spatial relationship: many X-class flares often lie at the center of the associated CME, while C-class flares widely spread to the outside of the CME span. The energy partition between flares and CMEs could be determined by their magnetic configration. The magnetic configuration of the C-class flare-CME events could have large variations, while that of the X-class events might be uniform, e.g. the CSHKP type configuration.

\section{Acknowledgements}

SOHO is a project of international cooperation between ESA and NASA. The LASCO data used here are produced by a consortium of the Naval Research Laboratory (USA), Max-Planck-Institut fuer Aeronomie (Germany), Laboratoire d'Astronomie (France), and the University of Birmingham (UK). Part of this effort was supported by NASA (NNX08AD60A).

\section{References}

Andrews, M. D. 2003, Solar Phys., 218, 261

Brueckner, G. E., et al. 1995, Solar Phys., 162, 357

Burkepile, J. T., et al. 2004, J. of Geophys. Res., 109, A03103

Jing, J., et al. 2005, Astrophys. J., 620, 1085

Gopalswamy, N. \& Thompson, B. J. 2000, JASTP, 62, 1457

Gopalswamy, N., et al. 2003, Astrophys. J., 586, 562

Harrison, R. A. 1986, Astron. Astrophys., 162, 283

Harrison, R. A. 1995, Astron. Astrophys., 304, 585

Harrison, R. A. \& Sime, D. G. 1989, Astron. Astrophys., 208, 274.

Harrison, R. A., et al. 1990, J. of Geophys. Res., 95, 917

Howard, R. A., et al. 1982, Astrophys. J., 263, L101

Hundhausen, A. J. 1997, AGU Monograph 99, 1

Kahler, S. W. 1992, ARAA, 30, 113

Kahler, S. W., et al. 1989, Astrophys. J., 344, 1026

Moon, Y.-J., et al. 2002, Astrophys. J., 581, 694

Munro, R. H. et al. 1979, Solar Phys., 61, 201

Veronig, A., et al. 2002, Astron. Astrophys., 382, 1070

Vršnak, et al. 2004, Solar Phys., 225, 355

Yashiro, S., et al. 2004, J. of Geophys. Res., 109, 7105

Yashiro, S., et al. 2005, J. of Geophys. Res., 110, A12S05

Yashiro, S., et al. 2006, Astrophys. J., 650, L143

Yashiro, S., et al. 2008, Astrophys. J., 673, 1174

Zhang, J., et al. 2001, Astrophys. J., 559, 452 


\section{Discussion}

WEBB: One can use LASCO halo CMEs to study the longitude distribution of flare sources. I have done this for some LASCO halos and found a distribution like you did.

YASHIRO: We have also analyzed the flare location distribution in the longitudinal direction using halo CMEs. The CME launch angle and width were estimated from a cone model. The preliminary analysis shows that may flares located at the center of the CME span, so the result is consistent with yours.

HOWARD: The distribution of "X" class flare site vs CME center looks to be more skewed than symmetric about " 0 " or co-located. The better correspondence of "X" vs "C" class flares could be a manifestation of the "big flare syndrome" of Gosling in that big events arise from a common instability.

YASHIRO: Not only in spatial relationship but also in energetic relationship, larger events show better correspondence between flares and CMEs. This might mean that the large events occur only in the CSHKP type magnetic configuration, while the small events occur in various configurations.

VRŠNAK: Spatial scatter of C-flares might be due to those events where flare only triggers the eruption of a meta-stable coronal structure (i.e. can be anywhere in the vicinity of the eruptive structure).

YASHIRO: I will check the largely-scattered C-class events with the point of view that you suggested.

MANDRINI: On the disk EUV images slow dimming regions, separated by a large distance. Depending on the location of the active region and orientation of the neutral line with respect to the limb, one can see that the flare may be at different distances from the two dimming regions. This may be responsible for the observed scatter.

YAshiro: Yes, EIT dimming extent might be used for the proxy of the CME span. It will be nice to examine the flare position with respect to the EIT dimming extent. 